\title{
Giambattista Marino, Virginio Orsini e Tommaso Melchiorri in materiali epistolari inediti e dimenticati*
}

Recenti esplorazioni del fondo Orsini (Fondo Orsini nell'Archivio Capitolino, Roma, serie I, corrispondenza epistolare di Virginio II Orsini= FO), custodito dagli inizi del secolo presso l'Archivio Capitolino, hanno portato all'individuazione di quattro lettere, grazie alle quali è possibile fare brevemente il punto sui rapporti tra Giambattista Marino e Virginio Orsini, ottavo duca di Bracciano, e sui loro contatti con Tommaso Melchiorri, secondo marchese di Turrita.

Ben poco è stato detto finora del legame che il letterato partenopeo ebbe con l'Orsini e la sua famiglia, vissuta tra la Toscana e Roma nei medesimi anni dei primi soggiomi nell'Urbe e dei primi viaggi del Marino.

$\mathrm{Da}$ un accenno contenuto in una lettera del poeta napoletano a Giambattista Manso, scritta a Napoli probabilmente nel 1594, secondo il quale il Marino avrebbe partecipato in veste di collaboratore e forse in quella d'autore ad una raccolta di "composizioni in lode della signora Peretta", raccolta poi naufragata, rimasta inedita o più semplicemente sconosciuta, è sembrato a Guglielminetti, curatore della più moderna e completa edizione di lettere mariniane, che questa opera sia stata concepita in onore di Flavia Peretti, moglie di Virginio dalla primavera del 1589 (Lettere [ed. Guglielminetti] 18). Nell'ultimo decennio del secolo il letterato partenopeo avrebbe così iniziato in patria a coltivare potenti amicizie romane; in effetti egli prestava attenzione, ad esempio, alle imprese militari del duca di Bracciano, in partenza per una campagna ungherese contro i Turchi, indirizzandogli un sonetto d'augurio che ebbe quasi sicuramente diffusione manoscritta. ${ }^{1}$ Eे difficile stabilire se egli rese anche omaggio alla duchessa, nipote di Sisto V, alla quale, così come è ricordato nel breve commento alla lettera ricordata (ibid.), era già stato dedicato il Tempio fabricato . . . , silloge poetica nata nel 1591 dalle cure di Torquato Tasso e di altri letterati; ${ }^{2}$ è invece ipotizzabile che il Marino lavorasse ad una nuova raccolta, forse ad un dono collettivo per Orsina Peretti, sorella di Flavia e sposa nel medesimo 1589 di Marcantonio Colonna, connestabile del Regno di Napoli e duca di Paliano.

Minime sono poi le indicazioni di contatti fornite nella biografia del letterato partenopeo composta alla fine del secolo scorso da Angelo Borzelli, ${ }^{3}$ secondo la 
quale il Marino al seguito del cardinale Pietro Aldobrandini fu tra il 1603 ed il 1605 anche all'ombra di altre potenti casate romane, quali ad esempio gli Sforza di Santa Fiora, i Peretti Montalto o appunto gli Orsini di Bracciano (Il cavalier 68). Gli studi eruditi dedicati al duca ed alla sua famiglia (si pensi ad esempio alle opere di Carlo Astolfi e di Vincenzo Celletti) hanno allo stesso modo ignorato il letterato ed i suoi omaggi a Virginio, con l'unica eccezione delle ricerche di Ferdinand Boyer, condotte non a caso in FO, grazie alle quali è stata frettolosamente stampata una lettera del poeta napoletano al duca del novembre del 1608 ("Virginio Orsini" 319), che non figura nella raccolta curata da Guglielminetti, né è ricordata nella nuova edizione della biografia mariniana del Borzelli.

Le quattro lettere autografe che ora si presentano, la prima e la quarta composte dal letterato partenopeo, le altre dedicate alle sue opere ed inviate da Marzio De Baschi e da Tommaso Melchiorri, tutte inedite ad eccezione dell'ultima che è appunto quella già proposta da Boyer ed è ora nuovamente trascritta dall'originale, costituiscono dunque il frutto di una sistematica ricerca nella corrispondenza del duca di Bracciano 4 .

Scritta il 28 maggio 1603 ed indirizzata all'Orsini, la prima è una missiva spedita dal poeta nel corso del suo terzo e più stabile soggiorno romano.

Inizialmente egli era giunto nella città per il giubileo del 1600 , forse al seguito di Fernando Ruiz de Castro, sesto conte di Lémos e viceré di Napoli, o in compagnia o per conto di Giambattista Manso (Borzelli, Il cavalier 46-47); ${ }^{5}$ poco dopo, in fuga dalle carceri della sua patria, vi aveva fatto ritorno dando principio ad una nuova, fruttuosa stagione della sua vita, scarsamente testimoniata dalla raccolta delle lettere mariniane (Lettere [ed. Guglielminetti] 27), durante la quale era entrato al servizio di Melchiorre Crescenzi (Borzelli, Il cavalier 51-52) ed aveva frequentato la bella dimora del chierico "presso la guglia del Sammoguto" ${ }^{\text {e }}$ quella di Onofrio Santacroce, dove ebbe sede una prima "Accademia Romana"?

Alla corte di suo zio, il granduca Ferdinando, Virginio Orsini negli stessi anni viveva con la sua famiglia tra le ville ed i palazzi fiorentini e pisani. Figlio di Paolo Giordano e di Isabella de' Medici, si era trasferito definitivamente in Toscana a partire dal giugno del 1595, abbandonando Roma dove, sposata la Peretti, aveva soggiornato in via di Parione ed aveva concepito i suoi primi figli. Dall'Urbe si era già allontanato nell'estate del 1594, per soccorrere Rodolfo II e, come si è scritto, per combattere contro i Turchi nelle terre ungheresi (Astolfi 14, 52-53); in seguito vi faceva forse sporadiche apparizioni, affidando definitivamente dalla metà del 1595 l'amministrazione del suo patrimonio romano ad alcuni collaboratori fidati.

Buone nuove inducevano frattanto il Marino a lasciare Roma per qualche tempo. Alla raccolta veneziana delle sue Rime era stata concessa licenza di stampa nel gennaio del 1601 (Rime, parte I, 22)) ed egli aveva così deciso di raggiungere la città lagunare per seguime le varie fasi di pubblicazione. Nell'autunno del 1601 si era fermato probabilmente a Firenze, da dove aveva scritto a Ga- 
spare Salviani delle "cortesie" di tanti gentiluomini della città (Lettere [ed. Guglielminetti] 28), ma è ipotizzabile che non ebbe modo allora di conoscere l'Orsini, poiché il duca in quel periodo era partito per la Spagna per cercare d'ottenere da Filippo III quella promessa del Toson d'oro, già concessa ai suoi avi ed ottenuta da Virginio tra la fine del 1601 e l'inizio dell'anno seguente (de Miranda, "Alessandro Tassoni" 46).

Nel febbraio del 1602 il Marino spediva da Venezia una lettera a Giambattista Strozzi, promettendo di tornare presto nella città medicea ed inviando saluti ossequiosi a Piero Strozzi, ad Alessandro Sertini ed a Baccio Gherardini, personalità e letterati che ben l'introducevano nell'ambiente fiorentino (Lettere [ed. Guglielminetti] 29). Un'altra testimonianza dei vivaci contatti con il medesimo mondo è inoltre in Borzelli, che ha ricordato l'amicizia del Marino con Giovanni Villifranchi, per molti anni corrispondente del duca di Bracciano così come appare da molte lettere in FO, e con Iacopo Corsi, del quale il letterato fu anche ospite (Borzelli, Il cavalier 54).

Al principio del medesimo 1602 Virginio tornava a Firenze, mentre il poeta licenziava le dediche delle due parti delle Rime, composte il 10 ed il 15 febbraio per il Crescenzi ed il Melchiorri (Rime, parte I, 3-15; parte II, 3-13), ed inseriva tra le "heroiche", le "morali" ed i sonetti di corrispondenza l'antica lirica per il duca, scritta in occasione della sua campagna ungherese, i versi per il Santacroce, per il Mattei e per il Villifranchi (parte I, 114, 133-34, 183, 243). Sollecitate la spedizione e la distribuzione dell'opera (Lettere [ed. Guglielminetti] 29), egli poi ripartiva alcuni mesi dopo per Roma, facendo sosta a Bologna (Delcomo 78 e 129), e tra la fine del 1602 e l'inizio del 1603 , nel suo secondo passaggio toscano, forse conosceva Virginio, mecenate e cultore di poesia.

Dal carteggio dei duchi di Bracciano appare chiaro quanto la loro vita fosse in quegli anni scandita da impegni di corte, da gioie e dolori familiari. Per la seconda metà del 1603 Flavia Peretti era infatti in attesa di un altro figlio ${ }^{8}$ ed una nuova gravidanza aveva l'anno seguente ${ }^{9}$ fino alla tragica nascita di un'ultima bambina, per la quale la duchessa moriva alla fine dell'estate del 1606 (Boyer, "L'infanzia" 242). Affidati alle cure di balie, istituzioni e tutori, la giovanissima prole degli Orsini cresceva allora in più luoghi ed in varie dimore, ${ }^{10}$ giungendo in tempi diversi anche a Roma.

Nel 1603 Virginio decideva di prendere parte ad un'altra spedizione contro i Turchi e per la nuova missione volle con sé il primogenito (Boyer, "Un cousin" 26-27; Celletti 138). Appena tredicenne, Paolo Giordano partiva così con il padre sulle galere dell'ordine di S. Stefano nei primi mesi dell' anno seguente, dopo una sosta nell'Urbe con il fratello Alessandro, che era a sua volta già indirizzato verso una brillante carriera ecclesiastica. ${ }^{11}$ Ed è in occasione del loro arrivo che si colloca probabilmente il primo recupero di un contatto epistolare tra il Marino, ormai noto per le sue Rime ed al centro di invidie incipienti, ${ }^{12}$ ed il duca di Bracciano. 


\section{Ill.mo et Ecc.mo Sig.re et p.ron mio oss.mo}

Poiche non posso con altro far conoscere al mondo la divota osservanza, ch'io porto alla casa di V.E., volsi questi mesi passati, nel tempo, che in Roma si aspettavano i due suoi Ill.mi figli, honorar la mia pen(n)a del lor nome, sforzandomi di adombrare il meglio, ch'io seppi, con due sonetti qualche raggio dell'una, et altra luce. Degnisi V.E. di gradire questa mia humile dimostratione d'affetto, di scusar l'ambitione, di perdonare all'ardimento, e di condonar questo, et quella al soprabondante desiderio, che vive in me di servire a V.E. A cui bacio reverentem(en)te le mani. Di Roma adì 28 di Maggio 1603

Di V.E.

Divotiss.o Ser.re

Gio(van) B(attist)a Marino. ${ }^{13}$

Accolte undici anni dopo ne La lira (parte III, 109), le due liriche auguravano al primo giovanetto gloria sui campi di battaglia, al secondo sacri onori. Con un altro componimento del letterato napoletano, scritto in seguito per la consegna ufficiale del Toson d'oro a Virginio (108), questi versi, come Borzelli ha sottolineato nella nuova edizione della sua biografia mariniana (Storia 83), furono riproposti dopo alcuni decenni dallo stesso Paolo Giordano nella sua unica raccolta poetica. Nelle stesse pagine fu anche aggiunto un sonetto di risposta, verosimilmente scritto dal primogenito di Virginio qualche anno dopo il 1603 (13841). Letterato più che condottiero, il nono duca di Bracciano li inserì tra i sonetti di corrispondenza, facendoli probabilmente trascrivere dalle antiche pagine manoscritte ricevute in dono dal poeta napoletano ed ignorando quelle varianti mariniane che furono evidentemente il frutto di una rielaborazione avvenuta in occasione della pubblicazione de La lira. ${ }^{14}$

Inviate a Virginio tra l'ottobre del 1603 ed il gennaio dell'anno seguente, le due lettere del De Baschi e del Melchiorri costituiscono una preziosa testimonianza dell'interesse orsiniano per le opere del Marino.

In quel periodo il letterato partenopeo passava al servizio del cardinale Pietro Aldobrandini, nipote di Clemente VIII, dedicando versi a buona parte della nobile famiglia del pontefice, al suo stesso protettore, che di volta in volta seguiva nelle residenze di Frascati, Subiaco e Tolfa (Borzelli, Il cavalier 57-61; Annibaldi, "Il mecenate (I)" 118, 149), ma piuttosto confuse sono per questo tempo le ulteriori notizie sulle relazioni romane del Marino fornite da Borzelli, secondo il quale, così come avveniva nei precedenti soggiorni, egli aveva ospitalità generosa nelle dimore delle più illustri famiglie di Roma, studiava e leggeva, ${ }^{15}$ stringendo inoltre amicizia con il cardinale Giambattista Deti (Il cavalier 67-71). ${ }^{16}$

Virginio risiedeva frattanto in Toscana, sempre attento alle opere di poeti, musicisti ed uomini di teatro ed alle attività di alcuni sodalizi accademici. ${ }^{17}$ Solo temporaneamente egli abbandonava la corte medicea per raggiungere a scopi terapeutici le fonti di S. Casciano nella provincia senese, ${ }^{18}$ forse proprio nei me- 
desimi giorni durante i quali a Roma il poeta napoletano aveva gravi problemi di salute (Lettere [ed. Guglielminetti] 38, 41, 43). ${ }^{19}$ Molteplici relazioni d'affari ma anche alcune informazioni letterarie gli giungevano continuamente dall'Urbe e dalla sua provincia.

Non era sfuggita al duca, così come attesta la lettera del suo collaboratore De Baschi che ora si presenta, la notizia della gestazione di un grande poema mariniano, già menzionato dal suo autore nella prima dedica delle Rime $^{20}$

\section{Ill.mo et Ecc.mo S.r P.ron mio Col.mo}

Vengo con questa à far riverenza a V. Ecc.za Ill.ma con darli conto ch'io sono à Roma, dove gli vivo al solito ser.re humiliss.mo, pregandola à farmi gratia di tenermi p(er) tale, con favorirmi alle volte di suoi comandi. L'altro giomo trovandomi con il S.r Marini gli dissi haver visto V. Ecc.za alli bagni, et che stava con desiderio aspettando veder una copia di qual che canto della sua opra, Me rispose che lui molto voluntieri servirebbe S. Ecc.za, ma che ha paura non se ne dia fuora copie, il che sarebbe con farlo morir impazzito, poi che lui hora la rivede, et la muta quasi tutta, et p(er) dirgliela liberam(en)te, lui dubita che il S.r Villafranchi non gli facci la burla. Io gli replicai che V. Ecc.za è Principe accuratiss(im)o, et ama sopra modo i virtuosi pari suoi, et che m'assicurano non ne lasserebbe pigliar copia à nessuno. Me ha pregato che gli ne scriva, $p(e r)$ haverne credo io parola da lei, poi che mi dice non haverne dato $\mathrm{p}(\mathrm{er})$ ancora nessuna copia fuori; Pero volendo V. Ecc.za esser servita, potra rispondermi, acciò gli la possi mostrare, et bisognando anco lasciare, et io procuraro che la venghi servita quanto prima, et di piu conti che sijno possibile, et $\mathrm{p}(\mathrm{er})$ fine recordandomeli al solito ser.re li bacio humilm(en)te le mani, et pregoli da Dio il colmo d'ogni felicità, Di Roma li 3 di 8 bre 1603

D(i) V. Ecc.za Ill.ma

Humiliss.mo et Devotiss.mo ser.re Martio de Baschi. ${ }^{21}$

L'opera, alla quale si fa riferimento, è con molte probabilità la Gierusalemme distrutta. Dopo la pubblicazione del 1602, i primi viaggi ed i numerosi omaggi ben distribuiti nelle corti frequentate, grande doveva essere infatti la curiosità per il nuovo cimento del letterato napoletano, ambiziosamente impegnato fin dall'inizio del secolo ${ }^{22}$ nella prova più ardua e gloriosa, quella cioè del poema.

$\mathrm{Al}$ di là del riferimento nella dedica al Crescenzi, ${ }^{23}$ suoi echi più tardi sono in molti sonetti della terza parte della Lira, ${ }^{24}$ nella premessa stessa del Claretti, secondo il quale, sebbene fosse "machina grande" ormai giunta a termine, necessitava di altro "tempo e sofferenza" (parte III, 65), ed in due lettere mariniane del 1616 e del 1620, la prima a Giacomo Castelvetro ${ }^{25}$ e la seconda al Ciotti (Lettere [ed. Guglielminetti] 257-59). Nessun'altra testimonianza precede o segue la pubblicazione dell'unico segmento conosciuto della Gierusalemme, il settimo canto, avvenuta un anno dopo la morte del poeta (Il settimo canto $1-47),{ }^{26} \mathrm{ma}$ il passaggio da una concreta fase progettuale dell'opera al suo accantonamento, coinciso probabilmente alla fine del primo quinquennio del XVII secolo con la 
lettura mariniana di Nonno Panopolita, è ben indicato nell'introduzione pozziana all'Adone (2.89, 2.91-93, 2.117-19) e rende più comprensibile il lavoro rapsodico del letterato partenopeo ${ }^{27}$ e l'inserimento successivo di alcune ottave del poema ne La strage de gl' innocenti (449-50; L'Adone 2.119).

Colpisce nella lettera del De Baschi l'attenzione con la quale il Marino proteggeva l'elaborazione piuttosto tormentata delle sue ottave, quel rischio di "morir impazzito" qualora si fosse giunti a copiare una parte o l'intero testo della Gierusalemme per imitarlo o diffonderlo senza la sua autorizzazione. Il legame con il Villifranchi, cortigiano ed emissario di Virginio, poteva rivelarsi pericoloso ma il poeta continuava ad essere particolarmente interessato all'ambiente fiorentino ed alle richieste dell'Orsini, lettore curioso e potente aristocratico, ed è per questo che, previa una garanzia scritta, sembrava disposto a superare gli indugi iniziali.

Non è noto al momento, se il duca scrisse poi la lettera d'assicurazione al letterato napoletano e se questi in effetti gli inviò alcune tranches manoscritte del suo poema. Stimolato dalla lettura di questi versi o incuriosito dalla difficoltà di ottenerli, Virginio si rivolse nei mesi seguenti anche al Melchiorri, nel tentativo di recuperare altre opere del poeta partenopeo.

Sui contatti tra il secondo marchese di Turrita ed il Marino si è detto finora poco, ma la vivacità del Melchiorri, "giovane elegante, simpatico, pieno di debiti, uno dei migliori viveurs" dell'Urbe, così come ha scritto Giovanni Nascimbeni, ${ }^{28}$ non doveva essere sfuggita al letterato napoletano, che gli faceva infatti omaggio nel 1602 della seconda parte delle Rime. Nella dedica, solo brevemente ricordata nella biografia di Borzelli (56), il marchese era definito un "chiarissimo ingegno, à bastanza di tutte le belle arti intendente, \& frà l'altre delle armoniche, come sono Musica, \& Poesia", un autore di "vaghissimi, \& dolcissimi componimenti, oltre à i gravi, e dotti discorsi recitati nella Romana Academia”, della quale, a dire del poeta partenopeo, era anche membro (Rime, parte II, 10-11). Mutevole si rivelava però nel tempo l'intensità delle lodi mariniane, se è vero che, nel riproporre dopo dodici anni la dedica, egli preferiva moderare gli elogi, passando da "chiarissimo" a "chiaro ingegno", da "vaghissimi, \& dolcissimi" a "vaghi, \& dolci componimenti", quasi avesse progressivamente mutato giudizio sul talento e sulle opere del Melchiorri ormai defunto (ibid.).

Testimonianza della loro amicizia giunge ancora da cinque sonetti presentati nella raccolta del 1602 (Rime, parte I, 136-37, 215, 251), grazie ai quali il letterato napoletano già si era probabilmente conquistato la protezione del Melchiorri ed aveva forse ottenuto il suo appoggio per introdursi nell'accademia del Santacroce e del Mattei. Nei medesimi anni è poi ipotizzabile che il marchese partecipasse alle tornate degli Umoristi (Maylender 5.375), ai quali fu anche legato il duca di Bracciano (Rossi 3.104-05), ma non è chiaro se mai in quel primo periodo di vita del sodalizio i due aristocratici intervennero per agevolare l'ingresso in accademia del Marino. ${ }^{29}$

Più antichi erano i legami tra Tommaso e l'Orsini, al quale ad esempio, già 
nel 1594, il futuro marchese manifestava totale fedeltà in occasione della menzionata campagna ungherese, apparsa agli occhi devoti del Melchiorri, "fra tanti rumori di guerra, rimbombo d'artiglierie, e strepito di armi", particolarmente avventurosa. ${ }^{30}$ Sebbene avesse sposato alla fine del 1596 una cugina di Virginio, Caterina Orsini del ramo dei signori di Vicovaro, ${ }^{31}$ ed appartenesse ad una famiglia conosciuta e ricca, il futuro marchese aveva con l'Orsini rapporti di comune cortigiano, curando una parte dei suoi interessi romani, per i quali era coinvolto anche Marcello Melchiorri, padre di Tommaso, ${ }^{32}$ ed inviandogli periodicamente saluti e doni. ${ }^{33}$ Troppo recente era la nobiltà dei marchesi di Turrita ${ }^{34}$ per potersi sottrarre ad un servizio, al quale piuttosto si prestavano con orgoglio, talvolta in collaborazione con Giovanni Brandano. ${ }^{35}$

Maggiore intimità rivelano comunque le lettere dedicate ad alcune preoccupazioni familiari, le brevi indicazioni ad esempio sulla salute di Caterina, colpita alla fine del 1603 da un ramo mentre era al sole "à darse la bionda à i capelli", ${ }^{36}$ ed è ipotizzabile che a cementare il legame tra Virginio ed il futuro marchese contribuissero anche comuni interessi letterari e musicali, ${ }^{37}$ come sembra confermare anche la comunicazione epistolare che qui è proposta.

\section{Ill.mo et Ecc.mo Sig.re et p.ron mio oss.mo}

Il Brandano mi hà comandato in nome di V.E., che io cerchi destramente haver alcuna compositione del Marino, ne sapendome dir quello, che V.E. più dessidera li mando una canzone di sospiri assai vaga, e due opre di quelle già date in luce, e in breve manderà similmente fuori un Polijnnio, cosi da lui chiamato dove in diverse canzonette di versi spezzati tratta molte cose curiose, e nuove, con altri sonetti e canzone indrizzandole al Sig.r Cardinale Aldobrandini, atte(n)dendo tuttavia al suo Poema, del quale non ho fatto istanza di haver parte alcuna, non sapendo il dessiderio di V.E. ben che in tutto non l'habbia tirato, ne ripolito. Fra tanto aspetterò, che la mi comandi in particulare, che farò ogni sforzo, che V.E. resti servita. La Sig.ra Caterina stà tuttavia male, ne si e visto fin hora altro meglioramento in lei, se non che mercoredì incominciò mover si poco la testa quanto si conosce che fa motivo. i medicamenti necessarij et espedienti al male non li hà voluti, ne vuole, tal che se Dio per miracolo non l'agiuta temo infinitamente che vi lassi la vita, che è quello mi occorre dire à V.E., alla quale facendo humilissima riverenza me le inchino. da Roma li 17 di Gennaro 1604.

D(i) V.E. Ill.ma

Humil.mo e Devot.mo Ser.re Tomaso Melchiori. ${ }^{38}$

Altra copia manoscritta della canzone dei sospiri il Marino inviava in quegli stessi mesi al Castello con una lettera d'accompagnamento (Lettere [ed. Guglielminetti] 32). Unitamente a Gli amori estivi, composti ancora dal letterato partenopeo, ai versi del Villifranchi e di altri autori, la lirica era pubblicata due anni dopo nella Corona di Apollo curata dal Gentile che, a testimonianza del consolidarsi del successo mariniano, definiva il trentacinquenne autore "poeta di 
quel grido ch'è noto a tutta Italia". 39

Non è chiaro poi quali potrebbero essere i parti letterari ai quali faceva riferimento il Melchiorri. La crescente fama dei componimenti del Marino e la citata pratica della loro diffusione, che prescinde dalla stampa, non facilitano l'individuazione di opere manoscritte. L'attuale conoscenza delle edizioni mariniane realizzate entro il gennaio del 1604 rende debole, d'altro canto, l'ipotesi dell'invio di testi stampati. ${ }^{40}$

Parimenti complessa è la successiva informazione letteraria, che costituisce la più antica notizia della Polinnia mariniana, poiché non è subito comprensibile se l'opera fosse composta unicamente da "canzonette di versi spezzati" o se includesse anche sonetti e canzoni.

A conferma della prima ipotesi giunge la testimonianza dello stesso autore con il tramite sia di Emanuele Filiberto Rovara, conte di Rovigliasco, nella dedica del Ritratto panegirico del novembre 1608 a Carlo Emanuele II di Savoia (10-11), sia di Onorato Claretti nella premessa sine data ai lettori della terza parte de La lira, dove l'opera inedita, "bella, piena di buona dottrina e varia", appariva nel 1614 esclusivamente composta da canzonette (56-57), già ribattezzate dal Rovara "hinni", senza dubbio più innovative e vicine, rispetto alle scelte metriche della seconda sezione delle Rime offerta al Melchiorri, all'accentuata sensibilità musicale del duca di Bracciano e del futuro marchese di Turrita. Delle canzonette della Polinnia il Marino ha poi scritto in una lettera parigina del 2 agosto 1619 a Santi Magnanini (Lettere [ed. Guglielminetti] 223), ${ }^{41}$ preceduta e seguita tra il medesimo 1619 ed il 1620 da brevi accenni alla raccolta in altre quattro lettere inviate dal poeta napoletano a Giambattista Ciotti (Lettere [ed. Guglielminetti] 212 e 258), a Lorenzo Scoto (214) ed ancora al Magnanini (224), nelle quali l'autore, conclusa da tempo la fase compositiva, dichiarava di star realizzando una copia della Polinnia per lo stampatore.

E probabile allora che il gruppo di sonetti e canzoni sia stato una sezione affiancata alla raccolta di "hinni", un'appendice aggiunta o una pubblicazione autonoma, che Tommaso annunciava come imminente, ma non è chiaro quale opera (la Polinnia, quest'ultima sezione o appendice, o entrambe) dovesse costituire all'inizio del 1604 l'omaggio più consistente per Pietro Aldobrandini.

Inedite e poi perdute, ad eccezione di qualche scheggia, le canzonette sono "riemerse" alla fine del secolo scorso per un'illusione di Borzelli, erroneamente convinto per alcuni anni d'averle ritrovate tra i manoscritti della Biblioteca della Societa Napoletana di Storia Patria; ${ }^{42}$ e pagine più recenti assicurano che la $P O-$ linnia, pur restando nell'ombra, non fu dimenticata dal Marino, divenendo in parte fonte d'ispirazione e laboratorio dal quale attinse l'autore per lo sviluppo di alcune sezioni dell'Adone (2.116-17).

L'ultima informazione riguarda ancora la Gierusalemme. Note propensioni orsiniane verso la poesia lirica e melica inducevano Tommaso, evidentemente ignaro della corrispondenza tra Virginio ed il De Baschi, a dubitare di un reale interesse del duca per l'epica mariniana. 
A quale scopo infine i componimenti mariniani erano stati "destramente" richiesti non è dato sapere. Si è ora detto che Virginio era un cultore delle lettere ed aveva grandi passioni musicali. E allora anche probabile che ricercasse le opere del poeta napoletano per farle musicare, così come facevano proprio in area toscana Marco da Gagliano, Giovanni Del Turco, Tommaso Pecci, Santi Orlandi e Francesco Bianciardi tra il 1602 ed il 1606 (Bianconi 9).

Rispetto alle precedenti l'ultima lettera è più tarda poiché è stata scritta, dal Marino, alla fine del 1608.

Sempre al servizio del cardinale Aldobrandini, il poeta, dopo un primo viaggio nel $1605,{ }^{43}$ si era trasferito l'anno seguente a Ravenna, da dove per incarichi e per diletto spesso si allontanava, soggiornando a Venezia, Bologna ed in altre città settentrionali (Borzelli, Il cavalier 74-79).

È di questo periodo la morte di Flavia Peretti, già in precedenza ricordata, e l'aggravarsi delle malattie di Virginio, definitivamente costretto a rinunciare a qualsiasi viaggio (Boyer, "Un cousin" 26). Di nuove missioni diplomatiche era così incaricato Paolo Giordano, al quale gradualmente passava anche la globale gestione del patrimonio orsiniano.

Nell'aprile del 1608 Pietro Aldobrandini ed il suo seguito giungevano a Torino, dove sostarono fino all'agosto del 1609 (Annibaldi, "Il mecenate (II)" 122), mentre in una precedente tappa genovese il Marino aveva modo di coltivare $\mathrm{i}$ suoi interessi pittorici, frequentando numerosi artisti e facendosi ritrarre da $\mathrm{Ca}$ stellino Castello (Giambonini 317).

A Roma, sui tavoli da gioco e sulle rive del Tevere, si consumavano frattanto gli ultimi, tragici giomi del Melchiorri, ben lontano ormai dall'immagine di letterato e "virtuoso" che il poeta napoletano aveva lasciato di lui nella dedica della seconda parte delle Rime (Marracci 446-50). Sopraggiunta il 22 aprile 1608, la morte del marchese di Turrita coglieva Virginio e la sua famiglia nel pieno dei preparativi per le nozze di Cosimo II e Maria Maddalena d'Austria (Boyer, "Un cousin" 29) ${ }^{44}$ e non lasciava invece tracce note nella raccolta di lettere o in altre opere del Marino che, pur distante, il poeta nell'autunno dello stesso anno volle ancora scrivere rendendo omaggio al duca di Bracciano, così come appare nell'ultimo recupero epistolare che è presentato.

\section{Ill.mo et Ecc.mo Sig.re et p.ron mio oss.mo}

Desideroso di ravvivare nella memoria di V.E. la ricordanza della mia divota servitù, piglio per occasione un poemetto da me nuovam(en)te composto, più per dimostrarmi in qualche parte grato a q(ue)sta Ser.ma Altezza, che per far pompa d'ingegno. È stato fatto in pochi giorni, poco riveduto, et con pochissima mia sodisf(atio)ne publicato. Il volume è picciolo, et di picciolo valore, ma l'affetto, che l'accompagna a V.E. è grande. Prendalo in segno della mia pro(n)ta volontà infino a tanto, che mi sia permesso dargliene testimonio piú chiaro. Et con tal fine li bacio 
humilmente le mani Di Torino adì 22 di Novembre 1608 .

Di V.E.

Divotiss.mo Ser.re

G. B. Marino. ${ }^{45}$

Il volume, già identificato da Boyer, era il menzionato Ritratto di Carlo Emanuele II di Savoia, freschissima princeps che non aveva però soddisfatto il Marino.

È comunque ipotizzabile che i contatti tra il poeta napoletano e l'Orsini non si esaurissero con quelli fin qui testimoniati.

A conclusione della prima sezione dei "ritratti", dedicata a prìncipi, capitani ed eroi, ne La galeria mariniana, che uscì a Venezia presso il Ciotti alla fine del 1619 , è infatti collocato un madrigale che ancora ricorda le antiche imprese guerresche di Virginio, ferito nella campagna ungherese del $1594 .{ }^{46}$ Non è facile stabilire una data per la genesi del suddetto gruppo di componimenti, ${ }^{47}$ ma noti sono gli inserimenti in altre sezioni di liriche composte per occasioni preceden$\mathrm{ti}^{48}{ }^{48}$ cosicché non è al momento accertabile quando la lirica è stata scritta o se mai è stata inviata al duca.

In compagnia di altri diciassette cavalieri romani, Virginio era infine menzionato dal letterato napoletano nella terza giornata dei giochi funebri in onore di Adone, con i quali si conclude il suo poema maggiore (20.314). ${ }^{49}$

Quando nel 1623 la princeps de L'Adone fu stampata da Oliviero di Varennes nella città francese, il duca si era ormai spento da otto anni e la sua presenza in quei versi, al fianco di numerosi aristocratici che all'epoca erano ancora in vita, costituiva quasi un'eccezione. Confusa nei brillanti colori dello stemma orsiniano, la breve apparizione di Virginio era un duplice omaggio alla memoria dell'uomo ed alla gloria del casato, le cui sorti ora dipendevano direttamente da Paolo Giordano e dai suoi numerosi fratelli.

\section{Università di Napoli}

\section{NOTE}

* Dedico a Giorgio Fulco con gratitudine queste pagine, che tanto devono ai suoi consigli preziosi. Ulteriori ringraziamenti vanno ai proff. Francesco Giambonini e Bruno Basile.

1 In FO non è traccia del componimento, né di lettere d'accompagnamento.

2 La data dell'opera è indicata unicamente nel colophon. Con maggiore precisione Angelo Solerti ha affermato che la gestazione del Tempio fabricato, concepito probabilmente a Roma in ambito accademico ed ivi stampato da Giovanni Martinelli, ebbe probabilmente inizio nel 1589 e si concluse nel dicembre del 1591 (634, 692-93).

3 All'edizione del 1927, pur "riveduta" e corredata da "molte illustrazioni nel testo e fuori testo" così come recita il frontespizio, si è quasi sempre preferita la princeps del 1898 , sostanzialmente più ricca e precisa.

4 Per la bibliografia del fondo si rinvia alle note di due miei saggi, dove sono pubblicate alcune lettere autografe rispettivamente di Tommaso Costo e di Alessandro Tassoni con- 
servate nel medesimo archivio. Al di là dell'acquisizione di altri materiali interessanti che saranno presto presentati, i sondaggi, che hanno consentito questi recuperi mariniani, smentiscono felicemente l'asserzione di Boyer secondo la quale la raccolta delle lettere di Virginio in FO "contiene una sola lettera di G.B. Marino" ("Virginio" 319).

5 Tanto il viaggio, quanto i rapporti del letterato con il Manso e la loro stessa corrispondenza sono stati più tardi messi totalmente in discussione dallo studioso, autore di una polemica monografia sul marchese di Villa (Giovan Batrista Manso 47-48, 75), alla quale con fiducia maggiore nelle fonti documentarie si è opposto Michele Manfredi, sostenendo l'ipotesi del soggiorno romano del Manso ed affermando, senza operare in sostanza alcun collegamento con gli spostamenti mariniani, che la loro diretta conoscenza doveva essere stata comunque precedente all'inizio del XVII secolo $(78-79,100-01)$.

6 La citazione, che ricorda la chiesa romana dei SS. Macuto, Alessandro e Bartolomeo acquistata nel 1539 dalla Confraternita dei Bergamaschi, è in una sua lettera veneziana del 1602 indirizzata a Tommaso Stigliani (Lettere [ed. Guglielminetti] 31).

7 In quel tempo egli compose tre sonetti per il Santacroce e per Mario Mattei, colonne portanti del sodalizio (Borzelli, Il cavalier 53).

8 Cfr. ad esempio la lettera d'auguri (Roma, 8 agosto 1603) di Camilla Peretti, sorella di Sisto V, scritta alla nipote Flavia, in FO, fascio 110 , volume III, lettera 586.

9 Come è indicato in una lettera di felicitazioni (Roma, 18 dicembre 1604) di Alessandro Damasceni Peretti, cardinale di Montalto e fratello di Flavia, spedita probabilmente al cognato Virginio, in FO, II, 362.

10 Cfr. la lettera (Roma, 6 agosto 1602) di Brigida Avvocati, inviata forse alla duchessa, in FO, 110, Il, 322, nella quale si parla della malattia di una piccola figlia degli Orsini, Isabella, ospite del monastero fiorentino di S. Apollonia. In seguito la bambina, con le sorelle Maria Felice e Camilla, era accolta in un altro monastero cittadino, quello della Santa Concezione (Boyer, "L’infanzia" 241-54).

11 Con il trasferimento definitivo in Toscana i due ragazzi, nati probabilmente a Roma (Astolfi 52), avevano forse avuto tra Siena e Pisa la loro prima educazione, ad integrazione della quale giungevano per l'appunto altri viaggi, esperienze nuove con il relativo intrecciarsi di conoscenze ed amicizie (Litta, vol. 8, tavola XXIX).

12 Si pensi ad esempio ai rapporti burrascosi, alle trascorse o coeve polemiche con il foggiano Giambattista Vitale, detto il Poetino (Marino, Poesie varie 413), e con il parmigiano Ferrante Carli (Delcomo 130).

13 Così come è avvenuto a gran parte delle lettere custodite in $\mathrm{FO}$, anche a questa, originariamente su un bifolio, è stata tagliata la seconda carta, sul verso della quale era probabilmente scritto il destinatario ed il suo indirizzo. È custodita in 113, IV, 492. Nel recto, in basso a sinistra, è comunque indicato, come segue, il destinatario: "Ill.mo et Ecc.mo S. Duca di Bracciano"; nulla è nel verso. Criteri conservativi sono stati utilizzati nella trascrizione di questa lettera, come delle altre che più avanti si presentano. Le abbreviazioni sono state però sciolte ad eccezione delle formule di ossequio. Mancano i due sonetti composti dal poeta per i figli del duca, trascritti probabilmente su un'altra carta, poi aggiunta alla lettera.

14 Grazie a queste correzioni, nel componimento dedicato a Paolo Giordano, la mano del giovinetto da "nata à lo scettro" diveniva "degna di scettro" (v. 8) ed il nemico, che prima reagiva al suo impeto guerresco celando addirittura "la testa", nascondeva poi unicamente "la destra" (v. 14); nei versi per Alessandro il cursus honorum del fanciullo, iniziato genericamente "in riva sacrata", nasceva con maggiore precisione e consapevolezza dei crescenti fasti romani del ragazzo "in riva del Tebro" (v. 3), e l'accenno all"'alta rugiada" della rosa, con la quale era metaforicamente indicato il valore temporale e spirituale dello stemma orsiniano, della famiglia tutta e dello stesso Alessandro, era scisso nella duplice immagine dell'"aura" profumata e della rugiada del nobile fiore (v. 11).

$15 \mathrm{Nel}$ rievocare ancora questi anni della vita del poeta, lo studioso ricordava il crescente in- 
teresse mariniano per la letteratura ecclesiastica e il successo di alcuni componimenti "spinti" di Girolamo Magagnati, diretti all’Orsini, a Michele Peretti, fratello di Flavia, e ad altri aristocratici, che furono probabilmente letti anche da alcune nobili dame (Storia 73-74).

16 Borzelli era convinto che il poeta napoletano già in quegli anni frequentasse nella dimora dell'alto prelato l'accademia degli Ordinati, ma parere diverso ha ad esempio avuto Giuseppe Gabrieli, per il quale l'istituzione guidata appunto dal Deti ed anche da Giulio Strozzi nasceva solamente nel 1608 (180).

17 Nella ricca corrispondenza del duca con letterati ed uomini di cultura della Toscana è a tal proposito significativa una lettera (Prato, 10 settembre 1603) di Cosimo Cicognini, breve segmento di un aggiornamento continuo sulle attività accademiche sollecitato dal duca stesso, in FO, 113, III, 334.

$18 \mathrm{Cfr}$. ad esempio la lettera (Roma, 2 agosto 1603) di Giambattista Acciaioli al duca in FO, 113, III, 322.

19 Come si sa nelle raccolte epistolari secentesche del Marino la data è soppressa. La proposta congetturale di datazione (in parentesi quadre) dell'edizione laterziana è stata ripresa con alcune rettifiche da Guglielminetti che, limitando ad un numero ridotto di casi l'uso delle parentesi quadre, sembra con quelle voler sottolineare la più problematica congetturalità di alcune datazioni. Così, in queste tre lettere romane si legge: “[1603]".

20 Scrivendo al Crescenzi, questi aveva infatti detto che la nascente creazione letteraria era fondata "sopra la vendetta della morte di Cristo, esseguita per divina volontà da Tito imperatore nella città di Gerusalemme" (Il settimo canto, parte I, 4-5).

21 La lettera è su un bifolio ed è conservata in FO, 113, II, 265. Le cc. 1v e $2 \mathrm{r}$ sono bianche; la c. $2 v$ ha invece in alto a sinistra l'indicazione e l'indirizzo del destinatario: "All'Ill.mo et Ecc.mo S.r P.ron mio Col.mo Il Sig.r mio Don Virginio Orsino Fiorenza".

22 Come attesta Giovanni Pozzi, l'aspirazione al "poema grande" ha condizionato buona parte della vita mariniana ( L'Adone 2.122-40).

23 L'accenno è stato ignorato da Angelo Colombo così da indurlo a credere ad una genesi molto posteriore della Gierusalemme ( 96 e 100-01).

24 Si pensi, tra tante liriche ivi dedicate al poema, a quelle del Pace Pasini e di Pietro Petracci $(335,338,359)$ o al componimento di Francesco Fresco $(336)$, riproposto in una terza edizione del Tebro festante (Marino, 1624, 3).

25 Edita per la prima volta in Butler, la lettera ricordava tra molte opere "distinte e finite" del letterato partenopeo anche "tre Poemi grandi" (553-54), cioè la Gierusalemme, l'Adone e le Trasformazioni, secondo l'autorevole proposta di Pozzi (L'Adone 2.158).

26 Di questa stampa, segnalata da Borzelli (Il cavalier 366) e descritta da Giorgio Fulco ("Bibliografia" 403-05), si è avvalso Pieri per un'edizione moderna della Gierusalemme (9-34).

27 Di altri canti inediti dell'opera ha parlato Fulco ("Bibliografia" 405), riprendendo un'informazione de Il ritratto del sonetto e della canzone di Federico Meninni (162).

28 Presentando due sonetti polemici degli inizi del Seicento, il primo composto da Antonio Caetani per mettere alla berlina Giulio Cesare Gonzaga, il secondo scritto da Alessandro Tassoni in difesa del suddetto aristocratico, lo studioso ha dato risalto ai molteplici interessi di Tommaso, che fu ad esempio abile giocatore di pillotta (Tassoni 1.7-10).

29 Sui rapporti tra il poeta partenopeo e gli Umoristi scrisse poi il Carli, assicurando che nel primo lustro del Seicento il Marino non aveva mai preso parte alle adunanze accademiche (Delcorno 130), ma la sua testimonianza non ha convinto tutti e Piera Russo, così come aveva già fatto Borzelli (Il cavalier 70-71), ha introdotto nel sodalizio il letterato napoletano già "nei primissimi anni del ' 600 " e gli ha addirittura attribuito, pur non rivelando la fonte della notizia, la carica di principe (60).

30 Cfr. la lettera (Roma, 2 settembre 1594) di Tommaso all'Orsini in FO, 112, II, 178: c. 1r.

31 Si veda una lettera (Roma, 28 dicembre 1596) del futuro marchese al duca di Bracciano in 
FO, 106, I, 94. Caterina era figlia di Giampaolo, cugino di primo grado di Paolo Giordano Orsini, padre di Virginio (Litta, vol. 8, tav. XXVII).

32 Cfr. la lettera (Roma, 17 giugno 1600) di quest'ultimo a Virginio in FO, 109, II, 462.

33 Per il viaggio francese, che nell'autunno del 1600 conduceva il duca alla corte dei Borbone in occasione delle nozze di Maria de' Medici e di Enrico IV, Tommaso ad esempio faceva preparare e spedire all'Orsini un abito di particolare eleganza, così come risulta da una sua lettera (Roma, 18 agosto 1600) a "Don Verginio" in FO, 109, I, 178: c. 1.r. Parallelamente l'Orsini mostrava di saper apprezzare gli omaggi del suo collaboratore, inviandogli in regalo, prima di partire poco dopo per la Spagna, un magnifico puledro. Cfr. la lettera di ringraziamento (Roma, 7 luglio 1601) in FO, 110, I, 48.

34 Accenni a Marcello Melchiorri, al suo acquisto del castello di Turrita e del titolo sono in Calcagni (368).

$35 \mathrm{Al}$ dottor Brandano, ennesimo emissario dei duchi di Bracciano, si fa ad esempio accenno in una lettera (Roma, 11 agosto 1601) di Marcello a Flavia Peretti in FO, 109, I, 51. Numerosi per questi anni sono i riferimenti al suo lavoro o le sue lettere nel suddetto fondo.

36 Nell'accorato messaggio (Roma, 10 gennaio 1604), inviato probabilmente a Virginio e custodito in FO, 114A, II, 367, Tommaso si lamentava della "sciocca vanità di Donne, che per parere, sono al fine di loro stesse homicide". Marcello aveva comunque già scritto al duca dell'incidente della Orsini in una lettera (Roma, 19 dicembre 1603) in FO, 110, III, 548, dove si faceva anche un accenno al Brandano.

37 Sulle passioni musicali di Virginio e di suo figlio Paolo Giordano, sul loro legame con i Caccini ed altri artisti e cantanti ha scritto nella prima metà del secolo Boyer ("Giulio"; "Les Orsini"). Più recentemente qualche accenno ai rapporti orsiniani con Luca Marenzio ed Emilio De' Cavalieri è nel lungo saggio di Claudio Annibaldi ("Il mecenate (I)" 37; "Il mecenate (II)" 101, 110), mentre sui loro contatti con Giambattista Signorini, Iacopo Pieri e Leonora Baroni ed ancora sulla loro relazione con Francesca Caccini si è soffermata Bianca Maria Antolini (349-50, 356, 359-60).

38 La lettera è custodita in FO, 114A, II, 414. Non è indicato il destinatario, né vi è traccia delle tre opere mariniane aggiunte a parte dal Melchiorri.

39 Per le canzoni del poeta napoletano, cfr. Della corona, parte I, 37-41; parte II, 5-11. La citazione dalla dedica è ancora nella parte I, 35.

40 Nell'ultimo decennio del XVI secolo alcuni versi del poeta partenopeo erano stati infatti accolti tra le rime di Giulio Cortese e di pochi altri letterati ma è piuttosto improbabile che nella lettera si accenni a tali pubblicazioni, così come al Prologo mariniano del Pastor fido di Battista Guarini che, edito sullo scorcio del Cinquecento, è custodito in un'unica copia in BNN (S.Q.XXV.L. 37 (11), segnalata da Croce (Poesie varie 403) e riproposta da Luigi Fassò (Guarini 274-82) e da altri studiosi. Inutilizzabile è anche la nuova curiosità bibliografica mariniana, emersa da una nota stiglianesca citata recentemente da Giulia Raboni (297), dove è accuratamente descritta una stampa partenopea della canzone dei baci, che fu vista forse dal letterato materano alla fine del XVI secolo, durante il suo soggiomo napoletano, poiché è difficile capire se il ricordo dello Stigliani sia un'ennesima mistificazione nella sua annosa querelle con il Marino o una testimonianza preziosa, che confutava quel breve passo della dedica al Melchiorri della seconda parte delle Rime, dove la canzone, ivi stampata per l'occasione (19-22), risultava ancora inedita nel febbraio del 1602 (5-7). Qui, nella princeps, il Marino considerava le indebite appropriazioni del componimento opera "di coloro, i quali non sape(n)do chi ne fusse l'auttore, l'hanno ò per errore, ò per altro a diversi falsame(n)te attribuita"; ma nelle ristampe, con minor astio e in maniera più ironica, opera "di coloro, i quali per vederla cosi errar vagabo(n)da, \& per non conoscere il suo leggittimo Padre, giudicandola orfana, la si hanno per pietà adottata" (7).

41 Per l'identificazione di Santi Magnanini, destinatario di alcune lettere parigine del lettera- 
to napoletano, erroneamente confuso con il più noto Ottavio, si veda De Maldé (125 n.69), nella quale la studiosa si è avvalsa di informazioni fornite da Fulco.

42 Alla sua proposta nel 1892 di pochi versi ed un indice della supposta opera mariniana seguiva sette anni dopo l'abiura.

43 Pur essendo suggerito, il soggiorno ravennate di quell'anno non è da Annibaldi espressamente documentato nella citazione della fonte ("Il mecenate (II)" 118, 156). Eloquenti sono invece due lettere del Marino scritte nella città romagnola, la prima al poeta fiorentino Simon Carlo Rondinelli nel febbraio 1605 e la seconda allo Stigliani più genericamente del 1605 (Lettere [ed. Guglielminetti] 49-52).

44 Sorte miglior toccava a Caterina Orsini che, superati gli antichi malanni, visse fino al 2 agosto 1617 (Litta, vol. 8, tav. VIII).

45 La lettera è custodita in FO, 118, II, 287. Nella c. 1r è indicato in basso a sinistra il destinatario: "Ill.mo Ecc.mo S.r Duca di Bracciano". La c. 1v è bianca. Manca il libro spedito.

46 Pubblicata da Marzio Pieri, l'edizione più moderna de La galeria è basata sulla seconda impressione, ancora realizzata a Venezia dal Ciotti, e minime sono le notizie che accompagnano il componimento per l'Orsini $(1.113,2.77)$.

47 Sulla questione si vedano inizialmente le note al testo di Pieri (1.XXXVIII), poi alcune considerazioni di Fulco ("Il sogno" 92).

48 Si pensi ad esempio al sonetto sulla Gerusalemme tassiana, inviato dal Marino a Bernardo Castello da Roma nel 1603 (Lettere [ed. Guglielminetti] 32) e collocato in seguito ne La galeria come terzultimo dei "capricci" pittorici (1.261).

49 Per la datazione e le intenzioni dell'ultimo canto, cfr. il commento di Pozzi (2.694-724). Si aggiungano ora sui medesimi versi le riflessioni di Francesco Guardiani ("I trastulli 303-04; "Il gran teatro").

\section{OPERE CITATE}

Annibaldi, Claudio. "Il mecenate 'politico'. Ancora sul patronato musicale del cardinale Pietro Aldobrandini (ca. 1571-1621) (I)". Studi musicali 16.1 (1987): 33-93.

"'Il mecenate 'politico'. Ancora sul patronato musicale del cardinale Pietro Aldobrandini (1571-1621) (II)". Studi musicali 17.1 (1988): 101-78.

Antolini, Bianca Maria. "Cantanti e letterati a Roma nella prima metà del Seicento: alcune osservazioni". In cantu et in sermone. For Nino Pirrotta on his 80 th birthday. Eds. Fabrizio Della Seta e Franco Piperno. Firenze: Olschki, 1989. 347-62.

Astolfi, Carlo. La presunta "casa di Sisto V" a via di Parione e le nozze di Flavia Peretti. Roma: Ferri, 1940.

Bianconi, Lorenzo. Il seicento. In Storia della musica. Vol. 4. Ed. Società Italiana di Musicologia. Torino: EDT, 1982.

Borzelli, Angelo. Di un manoscritto della Società Napoletana di Storia Patria falsamente attribuito al cavalier Marino. Noticina. Napoli: G. M. Priore, 1899.

. Giovan Battista Manso marchese di Villa. Napoli: Federico \& Ardia, 1916.

Il cavalier Giambattista Marino (1569-1625). Memoria premiata dall' Accademia

Pontaniana. Napoli: Gennaro M. Priore, 1898.

La Polinnia del cavalier Marino? Per le nozze del dottor Erasmo De Nuccio e di Maria Zona-De Nuccio. S. I., s. t., 1892.

- Storia della vita e delle opere di Giovan Battista Marino. Edizione riveduta con molte illustrazioni nel testo e fuori testo. Napoli: Tipografia degli Artigianelli, 1927.

Boyer, Ferdinand. "Giulio Caccini à la cour d'Henri IV (1604-5) d'après des lettres inédites". La revue musicale 7.11 (1926): 241-50.

"Les Orsini et les musiciens d'Italie au début du XVII siècle". Mélanges de philologie, d'histoire et de littérature offerts à Henri Hauvette. Paris: Les presses françaises 1934. 301-10. 
. "L'infanzia di Maria Felice Orsini a Firenze e il suo matrimonio con Enrico II di Montmorency (1599-1613)". Archivio storico italiano 121.438 (1963): 237-54.

. "Un cousin de Marie de Médicis en France: Virginio Orsini, duc de Bracciano".

Ausonia 8.1-4 (1943): 15-30.

. "Virginio Orsini ed i poeti del Secento (sic)". La cultura 5.7 (1926): 315-19.

Butler, Kathleen Theresa. "Two unpublished letters of Giambattista Marino". The Modern Language Review 31.4 (1936): 550-55.

Calcagni, Diego. Memorie istoriche della città di Recanati nella marca d'Ancona. Messina: Vittorino Maffei, 1711.

Celletti, Vincenzo. Gli Orsini di Bracciano. Roma: Palombi, 1963.

Colombo, Angelo. "Appunti sulla Gierusalemme distrutta. Vestigia di un insuccesso mariniano". Studi secenteschi 33 (1992): 93-134.

Delcorno, Carlo. "Un avversario del Marino: Ferrante Carli”. Studi secenteschi 16 (1975): 69-155.

Della corona di Apollo composta del più vago de' fiori di Permesso da Piergirolamo Gentile. Venezia: Sebastiano Combi, 1605.

De Maldé, Vania. “Sull'ortografia del Seicento: il caso Marino". Studi di grammatica italiana 12 (1983): 107-66.

de Miranda, Girolamo. "Alessandro Tassoni tra Virginio Orsini ed Ascanio Colonna (con tre lettere inedite)". Filologia e critica, 17.1 (1992): 44-51.

. "Due lettere inedite di Tommaso Costo". Esperienze letterarie 17.4 (1992): 41-62.

Fulco, Giorgio. "Bibliografia mariniana sommersa". Filologia e critica 3.2-3 (1978): 400-12.

. "Il sogno di una «galeria»: nuovi documenti sul Marino collezionista". Antologia di belle arti 3.9/12 (1979): 84-99.

Gabrieli, Giuseppe. "Gli Umoristi". Roma 13.4 (1935): 173-84.

Giambonini, Francesco. "Cinque lettere ignote del Marino". Forme e vicende per Giovanni Pozzi.

Eds. Ottavio Besomi, Giulia Gianella, Alessandro Martini e Guido Pedrojetta. Padova: Antenore, 1988. 307-30.

Guardiani, Francesco. "Il gran teatro del mondo, ovvero il mondo a teatro". Lectura Marini. Ed. Francesco Guardiani. Ottawa: Dovehouse, 1989. 325-40.

. "I trastulli del cinghiale". Lectura Marini. Ed. Francesco Guardiani. Ottawa:

Dovehouse, 1989. 301-16.

Guarini, Battista. Opere. Ed. Luigi Fassò. Torino: Utet, 1950.

Litta, Pompeo. Famiglie celebri d' Italia. 13 voll. Milano: Paolo Emilio Giusti, 1819-83.

Manfredi, Michele. Gio. Battista Manso nella vita e nelle opere. Napoli: Jovene, 1919.

Marino, Giambattista. L'Adone. Ed. G. Pozzi. 2 voll. Milano: Mondadori, 1976.

1960.

Dicerie sacre. La strage de gl'innocenti. Ed. Giovanni Pozzi. Torino: Einaudi,

. La galeria. Ed. Marzio Pieri. 2 voll. Padova: Liviana, 1979.
Gierusalemme distrutta e altri teatri di guerra. Ed. M. Pieri. Parma: La Pilotta,
. Lettere. Ed. Marziano Guglielminetti. Torino: Einaudi, 1966.
Lettere. Eds. Angelo Borzelli e Fausto Nicolini. 2 voll. Bari: Laterza, 1911-12.
La lira. Venezia: G.B. Ciotti, 1614.
. Poesie varie. Ed. Benedetto Croce. Bari: Laterza, 1913.
Prologo sopra del Pastor Fido, rappresentato nella città di Nola l'anno 1599. S.

1.: s. t., 1599 .

. Rime. Venezia: Giambattista Ciotti, 1602.

Il ritratto del serenissimo don Carlo Emanuello, duca di Savoia, panegirico.

Torino: s. t., 1608.

. Il settimo canto della Gierusalemme distrutta. Venezia: Girolamo Piuti, 1626.

Il Tebro festante. Venezia: Ciotti, 1624.

Marracci, Ippolito. Principes Mariani, seu, de praecipuis christianorum principum, infra caesares 
regesque, in Mariam Deiparam Virginem obsequijs, liber unus. Roma: Ignazio De Lazzaro, 1660.

Maylender, Michele. Storia delle accademie d'Italia. 5 voll. Bologna: Cappelli, 1926-30.

Meninni, Federico. Il ritratto del sonetto e della canzone, discorsi. Napoli: Giacinto Passaro, 1677.

Orsini, Paolo Giordano. Rime. Bracciano: Andrea Fei, 1648.

Raboni, Giulia. "Geografie mariniane. Note e discussione sulle biografie seicentesche del Marino". Rivista di letteratura italiana 9.1-2 (1991): 295-311.

Rossi, Giovan Vittorio [Eritreo, Giano Nicio]. Eudemia. Leyda: s. t., 1637.

Russo, Piera. "L'accademia degli Umoristi. Fondazione, strutture e leggi: il primo decennio di attività". Esperienze letterarie 4.4 (1979): 47-61.

Solerti, Angelo. Vita di Torquato Tasso. Vol. 1. La vita. Torino-Roma: Loescher, 1895.

Tassoni, Alessandro. Opere minori. Eds. Giovanni Nascimbeni e Giorgio Rossi. 3 voll. Roma: Formiggini, 1926.

Tempio fabricato da diversi coltissimi, e nobiliss. ingegni, in lode dell' illustr.ma e ecc.ma donna Flavia Peretta Orsina, duchessa di Bracciano. Dedicatole da Uranio Fenice [Torquato Tasso]. Roma: Giovanni Martinelli, 1591. 